\title{
Model for Melt Fracture Instabilities in the Capillary Flow of Polymer Melts
}

\author{
Joel D. Shore, ${ }^{1}$ David Ronis, ${ }^{1,2}$ Luc Piché, ${ }^{1,3}$ and Martin Grant ${ }^{1}$ \\ ${ }^{1}$ Department of Physics and Centre for the Physics of Materials, McGill University, \\ 3600 University Street, Montréal, Québec, Canada H3A 2T8 \\ ${ }^{2}$ Department of Chemistry, McGill University, 801 Sherbrooke Street West, Montréal, Québec, Canada H3A 2 K6 \\ ${ }^{3}$ Industrial Materials Institute, National Research Council of Canada, \\ 75 De Mortagne Boulevard, Boucherville, Québec, Canada J4B $6 Y 4$
}

(Received 26 December 1995)

\begin{abstract}
We model the capillary flow of a polymer melt, incorporating a stick-slip boundary condition at the wall. The boundary condition is enforced by a phase-field model for the local state of the polymer, which describes the kinetics of a first-order transition. We numerically solve the linearized NavierStokes equations, coupled to this prescribed boundary condition and to a Maxwell model for viscoelasticity. In various regimes, the model exhibits steady flow, periodic oscillations, and more complicated spatiotemporal structures, which can be observed experimentally. [S0031-9007(96)00756-9]
\end{abstract}

PACS numbers: 61.25.Hq, 47.50.+d, 61.41.+e, 64.60.My

The capillary flow of molten polymers has received much attention in the plastics and chemical engineering communities $[1,2]$ because at higher flow rates the extrusion of the polymer melt is commonly accompanied by instabilities which manifest themselves as surface distortions, called "melt fracture," in the final plastic product. A standard experiment has the melt pushed from a large reservoir into the capillary ("die") and extruded out the other end. Typically, as the flow rate is increased, the extrudate first develops a fine-scaled sawtooth texturing on its surface called sharkskin; next, for experiments performed at a constant flow rate into the reservoir (as opposed to constant pressure), there are relatively long timescale regular undulations during what is called "spurt" flow; finally, a very disordered lumpy structure called "gross melt fracture" is observed at the highest flow rates. It is controversial whether these effects are due to processes inside the die or are instead effects occurring at the entrance or exit of the die.

The specific motivation for the model we will introduce is recent work which implies that polymeric fluids might not always obey "stick" boundary conditions on mesoscopic length scales. In particular, de Gennes and co-workers [3] have suggested that polymer melts can slip at walls and, moreover, that a sharp transition between slip and stick should be observed as the shear rate at the walls is increased. Indeed there is experimental evidence for the existence of slip in polymer melts. While much of this evidence has been rather indirect $[1,2,4]$, a recent experiment by Migler et al. [5] measured the velocity of a polymeric fluid within $100 \mathrm{~nm}$ of the wall and found a sharp transition between small and large slip velocities as the shear rate was increased. A further impetus for our work is recent ultrasonic measurements [6], which show that anomalous time-dependent behavior in the polymer flow occurs within the die, far from both the entrance and exit, suggesting that instabilities occur inside the die itself.
Here we generalize these ideas by introducing a hydrodynamic model to describe the flow of a viscoelastic fluid in which the conformation of polymers near the surface of the die undergoes a first-order transition as a function of the shear stress at the wall. This conformational change leads to a change in the frictional force between the wall and the polymer in the bulk, producing stick-slip behavior and leading, in a natural way, to a multivalued flow curve (Fig. 1); such a curve has been prevalent in the recent literature $[1,2]$. When the die is coupled to a large reservoir in which the polymer can be compressed, we find that the multivalued flow curve gives rise to oscillatory spurt flow as has been discussed previously [2]. More significantly, and even in the absence of the reservoir, we find that the elastic nature of the fluid can give rise to periodic oscillations, chaotic behavior, and large-scale spatial structures in the die, which we conjecture is responsible for sharkskin.

The slipping length, $\ell \psi(\mathbf{x}, t)$, is commonly defined by a relation between the velocity component parallel to the wall, $\mathbf{v}_{\|}$, and its normal derivative [1,3-5]; i.e.,

$$
\mathbf{v}_{\|}=\ell \psi \hat{\mathbf{n}} \cdot \nabla \mathbf{v}_{\|},
$$

with $\hat{\mathbf{n}}$ the inward unit normal. Here $\ell$ is a constant and $\psi$ is a dimensionless quantity defined locally at each point $\mathbf{x}$ along the walls at time $t . \psi=0$ gives stick, while $\psi \rightarrow \infty$ gives total slip. Experiments show that the slipping length jumps sharply at a critical value of the shear [4,5], while hysteresis is observed on the multivalued flow curves seen in extrusion [1,2]. Together, these two features suggest the change in slipping length is the nonequilibrium analog of a first-order phase transition, presumably the result of a transition in the local conformational state of the polymer at the wall.

We describe this using a Ginzburg-Landau phase-field model for $\psi(\mathbf{x}, t)$, as is standard in the theory of phase transitions $[7,8]$. The microscopic picture of de Gennes and co-workers [3] suggests associating larger $\psi$ with the stretching and uncoiling of some polymers attached to the 


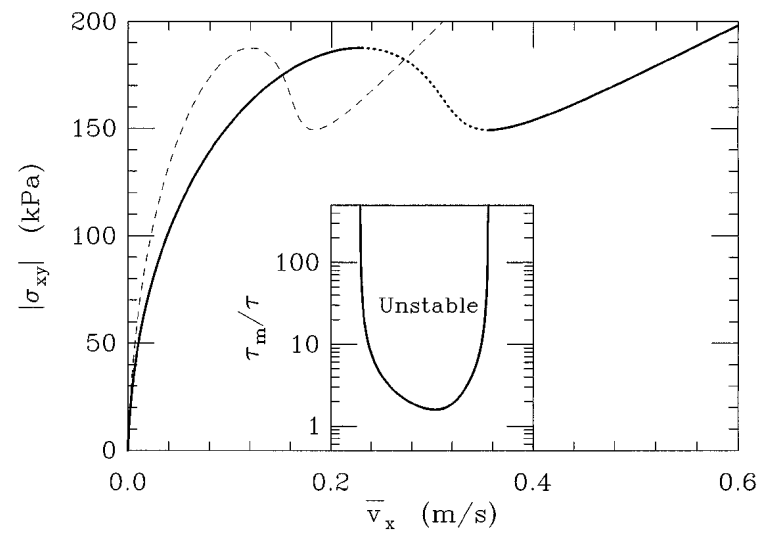

FIG. 1. Flow curve, showing the magnitude of the shear stress at the wall $\left|\sigma_{x y}\right|$ vs the average flow velocity $\bar{v}_{x}$ in the steady state. A plot with $\bar{v}_{x}$ replaced by the slipping length $\ell \psi$ looks qualitatively similar. The dotted part of curve has $\bar{\chi}^{-1}<0$ and is potentially unstable; the solid part is stable. The inset shows the range of $\bar{v}_{x}$ where the steady-state solution is actually linearly unstable as a function of $\tau_{m} / \tau$, as obtained from Eq. (4). Thin dashed line shows flow curve obtained if $\ell$ is reduced by a factor of two; see text for discussion.

wall although other mechanisms, such as absorption or desorption, have also been proposed as the cause of the stick-slip transition in some systems [4]. The first-order transition is determined by the free energy functional

$$
F=\int_{\text {walls }} d \mathbf{x}\left[\left(\xi_{0}^{2} / 2\right)(\partial \psi / \partial \mathbf{x})^{2}+f(\psi)\right],
$$

where the local free energy density is $f(\psi) \equiv(c / 4) \psi^{4}-$ $(b / 3) \psi^{3}+(a / 2) \psi^{2}-H\left|\sigma_{x y}\right| \psi, \sigma_{x y}$ is the local shear stress at the wall, and $a, b, c, H$, and the bare correlation length $\xi_{0}$ are positive constants. The time dependence of the process obeys [7]

$$
\tau \partial \psi / \partial t=-\delta F / \delta \psi
$$

where $\tau$ is a kinetic coefficient related to the time scale for polymer reorientation.

The equilibrium value of $\psi$ is zero for $\sigma_{x y}=0$, but as the stress increases, for appropriately chosen values of the parameters, there can be a first-order transition to a large value of $\psi$. Since there is a range of values of $\left|\sigma_{x y}\right|$ where the local free energy density $f(\psi)$ has a doublewelled structure, this gives rise to hysteresis associated with the spinodal part of the van der Waals loop in $\partial f / \partial \psi$, where the local susceptibility $\chi \equiv\left(\partial^{2} f / \partial \psi^{2}\right)^{-1}$ is negative, while retaining consistency with a sharp firstorder phase transition in the thermodynamic limit. This hysteresis has been seen experimentally, and much recent theoretical work has incorporated it $[1,2,9]$.

To complete the description of the fluid, the boundary condition (1) must be coupled to the bulk fluid flow. We simply use the linearized Navier-Stokes equation for an incompressible fluid, with Maxwell's constitutive relation for the viscous stress of a viscoelastic fluid [10]. Namely, the velocity field $\mathbf{v}(\mathbf{r}, t)$ at position $\mathbf{r}$ satisfies $\rho \partial \mathbf{v}(\mathbf{r}, t) / \partial t=-\mathrm{A} \nabla p(\mathbf{r}, t)+\nabla \cdot \overleftrightarrow{\sigma}(\mathbf{r}, t)$. Here $\rho$ is the density of the melt, assumed incompressible $[\nabla$. $\mathbf{v}(\mathbf{r}, t)=0$ ], and $p$ is pressure. The viscous stress tensor $\stackrel{\leftrightarrow}{\sigma}$ satisfies [10] the Maxwell model $\tau_{m} \partial \overleftrightarrow{\sigma}(\mathbf{r}, t) / \partial t=$ $-[\stackrel{\leftrightarrow}{\sigma}(\mathbf{r}, t)-\eta \overleftrightarrow{\varphi}(\mathbf{r}, t)]$, where the rate of strain tensor components are $e_{i j} \equiv \partial v_{i} / \partial x_{j}+\partial v_{j} / \partial x_{i}$. Depending on the Maxwell relaxation time $\tau_{m}$, this interpolates between a viscous fluid $\left(\tau_{m} \rightarrow 0\right)$ with viscosity $\eta$ and an elastic solid $\left(\tau_{m}, \eta \rightarrow \infty\right)$. This constitutes a minimal model necessary to demonstrate our results. Neglecting the nonlinear convective term in the Navier-Stokes equation is an excellent approximation, while estimates suggest that the convective terms in the Maxwell relation are small for the range of flow rates considered [11]. Some limited numerical work incorporating shear-thinning [10] suggests it does not qualitatively alter our basic picture.

Hereafter, we shall consider a two-dimensional slit of size $L_{x} \times L_{y}$ with periodic boundary conditions in the $x$ (flow) direction and impermeable walls at $y=0$ and $L_{y}$ in the direction normal to the flow. It is convenient to separate the pressure into a uniform pressure gradient and an excess part, as $p(\mathbf{r}, t)=g(t) x+\delta p(\mathbf{r}, t)$. The excess part $\delta p(\mathbf{r}, t)$ is periodic in $x$, while the uniform gradient satisfies $g(t)=\left[\bar{\sigma}_{x y}\left(L_{y}, t\right)-\bar{\sigma}_{x y}(0, t)\right] / L_{y}$, where $\bar{\sigma}_{x y}(y, t)$ is the average of the shear stress over the flow direction $x$. This enforces a constant flow rate through the die, which we find to be necessary to produce sustained oscillations when the boundary conditions are uniform along the entire wall. (Relaxation of this condition in the nonuniform case is discussed below.)

We use material parameters typical of commodity polymers: $\rho=740 \mathrm{~kg} / \mathrm{m}^{3}$ and $\eta=1.0 \times 10^{4} \mathrm{Pas}$. We choose a capillary size $L_{y}=4 \times 10^{-3} \mathrm{~m}$. The parameters in the phase-field model are taken to be $c=10$, $b=13, a=5, H=3.2 \times 10^{-6} \mathrm{~Pa}^{-1}$, and $\ell=4 \times$ $10^{-2} \mathrm{~m}$. These values are chosen so that steady-state solutions of the equations give a multivalued flow curve (Fig. 1) typical of ones seen experimentally [1,2]. The three branches of the curve in a range of $\left|\sigma_{x y}\right|$ correspond directly to the two minima and one maxima in $f(\psi)$. Several authors have suggested that the negative slope regime of such a flow curve should lead to unstable flow $[1,2,9]$. We will investigate the model over various ranges of the phenomenological parameters $\tau, \tau_{m}$, and $\xi_{0}$, the slit length $L_{x}$, and the average flow velocity $\bar{v}_{x}$.

The range of $\bar{v}_{x}$ for which oscillations occur can be determined from an exact linear stability analysis about the steady-state value $\psi=\bar{\psi}$ [12]. To a good approximation, the criterion for instability of the steady state is simply

$$
\bar{\chi}^{-1}+\xi_{0}^{2} k_{x}^{2}<-\tau / \tau_{m},
$$

where $\bar{\chi}^{-1} \equiv a-2 b \bar{\psi}+3 c \bar{\psi}^{2}$. We see that the most unstable mode has wave number $k_{x}=0$. It can be shown that $\bar{\chi}^{-1}$ is negative precisely on that part of the flow curve between the maximum and the minimum, indicated by the dotted portion of the curve in Fig. 1. Thus (4) will be satisfied for $k_{x}=0$ over a range of $\bar{v}_{x}$, provided that $\tau_{m} / \tau$ is sufficiently large. We shall call this range the stickslip regime. The kinetics of the first-order transition from 


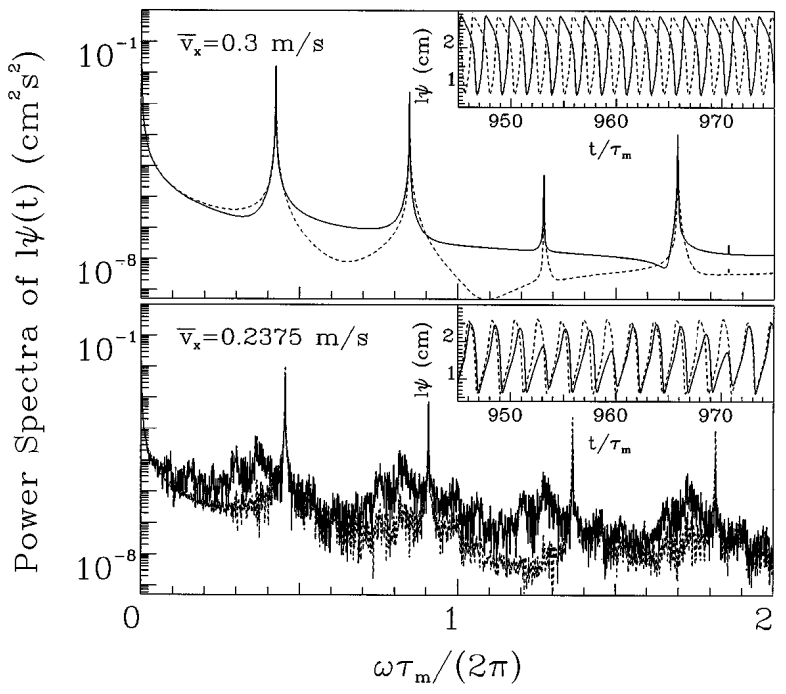

FIG. 2. Behavior of the slipping length $\ell \psi$ at the two walls for $\xi_{0} \gtrsim L_{x}$. Shown are power spectra of $\ell \psi(t)$ for regular oscillations (top) and chaos (bottom); insets show time traces. Walls at $y=0$ and $L_{y}$ are indicated by solid and dashed lines, respectively. Parameters used are $\tau_{m}=10^{-3} \mathrm{~s}, \tau=10^{-4} \mathrm{~s}$; the exact values of $\xi_{0}$ and $L_{x}$ are not relevant.

small $\psi$ ("stick") to large $\psi$ ("slip") are the origin of oscillations in this regime: At a critical stress the stick state becomes metastable, and at some stress beyond that the system jumps to the slip condition. However, once in the new slip state, the stress between the fluid and wall decreases, the slip state itself becomes metastable, and a transition to stick eventually ensues. Hence the system can repeatedly cycle between stick and slip. This is similar to the mechanism giving oscillations in the FitzHugh-Nagumo model [13]. However, unlike the normal spinodal instability for which $\bar{\chi}^{-1}<0$, here the criterion for instability also depends on the relative magnitudes of the elastic time scale, $\tau_{m}$, and the time scale for relaxation of the conformation of the polymer at the surface, $\tau$, as shown in the inset to Fig. 1. If $\tau_{m} / \tau$ is too small, there is no instability; the physics is essentially that of two coupled oscillators (the wall and the fluid in the bulk), and if $\tau_{m}$ is too small the system is overdamped. Thus the elastic nature of the fluid (a large $\tau_{m}$ ) is an essential ingredient of the instability.

We have carried out further investigation of our model numerically, with a mixed finite-difference and spectral method. First, we analytically Fourier transformed the flow equations to obtain an infinite set of coupled ordinary differential equations, which were reduced to a finite number by neglecting high wave numbers. They, along with the nonlinear boundary condition, can then be propagated forward in time by standard methods [14]. For the one-dimensional case, where the system is assumed to be uniform in the flow direction, we have verified that this method gives the same results as solving in real space by finite differences.

We first consider the limit $\xi_{0} \gtrsim L_{x}$ [15], where the system is uniform in the flow direction. The results from the linear stability analysis are confirmed by the numerics; in particular, the stability criteria for $k_{x}=0$ is in excellent agreement with where oscillating solutions first occur numerically. It is also found that the period of those oscillations is $\mathcal{O}\left(\tau_{m}\right)$, as is evident in Fig. 2. An orderof-magnitude estimate of the $\tau_{m}$ appropriate for polymer melts yields an oscillation period which corresponds well, given the flow rate, to the wavelength of experimentally observed sharkskin [12]. For values of the average flow $\bar{v}_{x}$ which are close to the minimal and maximal ranges of the stick-slip regime, we find complex oscillatory behavior. This includes chaos [16], shown in Fig. 2, as well as oscillations with periodicities that are integer multiples of the fundamental oscillation period. The oscillations of $\psi$ at the two walls are most often out of phase, although oscillations still occur if mirror symmetry is enforced.

For smaller $\xi_{0}$, rolls spanning the capillary can appear, Fig. 3(a); these rolls are coupled to the out-of-phase oscillations at the walls [17]. Indeed, experimentally, sharkskin in a cylindrical die often has a spiral pattern with opposite sides of the extrudate being out of phase. In our model, the roll structures in Fig. 3(a) lead to complicated spatiotemporal patterns at the walls, Fig. 3(b). These, and analogous structures in the third dimension not included in our calculation, should manifest themselves as defects on the surface of the extrudate. Although no direct comparison can be made without including the third dimension, we are encouraged by recent experiments [6] showing

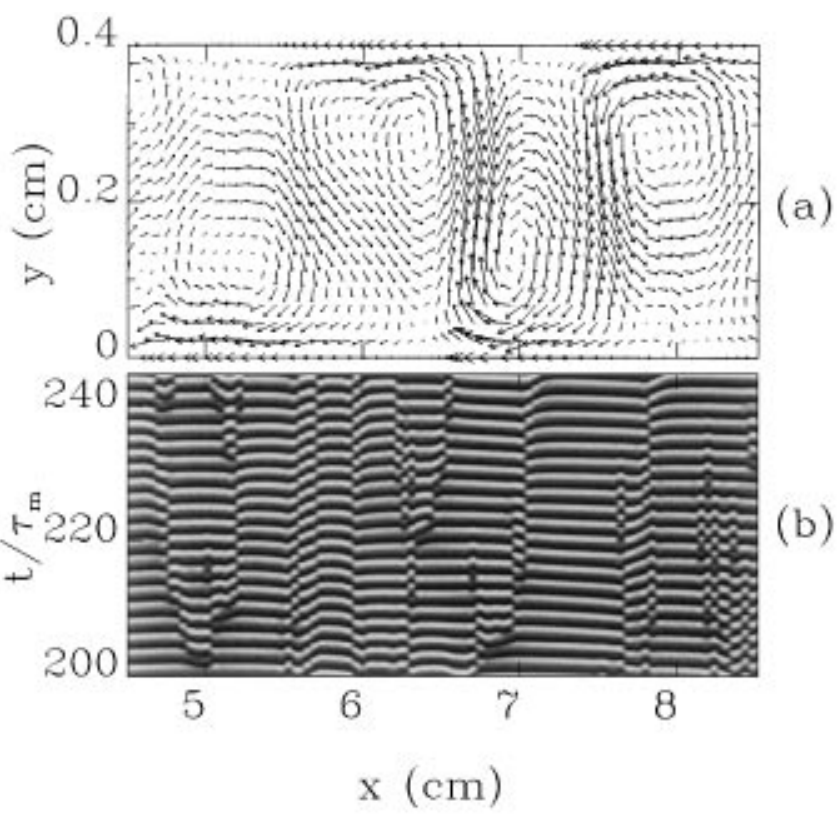

FIG. 3. (a) Typical random flow pattern in the channel for $\xi_{0} \ll L_{x}$. Shown is the velocity relative to the mean flow velocity, $\mathbf{v}(x, y)-\overline{\boldsymbol{v}}_{x} \hat{\mathbf{x}}$, with the magnitude indicated by the size of the arrow. (b) Space-time plot of the slipping length $\ell \psi(x, t)$ along one of the channel walls. The value of $\ell \psi$ is indicated by the brightness on this gray scale plot. The defect structures evident in (b) are associated with the rolls in the channel shown in (a). Parameters used are the same as in the bottom panel of Fig. 2, except here $\xi_{0}=0 \mathrm{~m}$ and $L_{x}=0.1 \mathrm{~m}$. 
defect patterns on the surface of the extrudate similar to the patterns seen here in the $x$ - $t$ plane.

In order to make closer contact with experiments, two more features must be included in our model. The first is motivated by recent work $[1,2]$ showing that the long timescale oscillations seen in the spurt regime are controlled by the volume and compressibility of the polymer in the reservoir. This extrinsic effect is easily incorporated by considering the reservoir roughly as a spring [2,12], which gives us one additional second-order differential equation for the average fluid velocity in the die. We then see "reservoir compressibility oscillations" which have the expected linear behavior on the volume of polymer in the reservoir.

As has already been alluded to, we conjecture that the viscoelastic oscillations in our model account for the sharkskin distortions seen in the extrudate. However, when included, reservoir compressibility oscillations become the controlling phenomenon, since the polymer in the die spends very little time at a flow rate in the stickslip regime, and thus viscoelastic oscillations do not get excited. In order to recover the possibility of viscoelastic oscillations, we must include the fact that die is not uniform and, in particular, that full solutions of the fluid flow equations with realistic constitutive laws show that higher stresses are present near the exit of the die [1]. We can do this most simply by assuming that a portion ("end") of the die has a smaller slip length coefficient, $\ell$, cf. Eq. (1), and thus the flow curve for that region is shifted to the left of the primary flow curve (see Fig. 1). For example, we assume that $20 \%$ of the die has an $\ell$ half that is in the remainder of the die. This allows us to reproduce all the important features seen in experiments. For example, we find that there can be viscoelastic oscillations in the end region of the die even when the system has not yet reached the stick-slip regime of the primary flow curve and these oscillations are now sustained, whether or not the polymer is pushed into the reservoir at constant flow rate or constant pressure, in agreement with experimental observations of sharkskin [2]. Furthermore, when the system is run at a constant flow rate in the stick-slip regime of the primary flow curve, we see reservoir compressibility oscillations (spurt flow) throughout the die, with viscoelastic oscillations superimposed in the end of the die during part of the stick portion of the cycle. Indeed, experimentally, sharkskin is frequently observed on this part of the extrudate in the spurt flow regime.

In summary, we have presented a model for the melt fracture instabilities seen in the extrusion of polymer melts; it includes a first-order transition between stick and slip behavior of the polymer at the walls as a function of the shear stress. The incorporation of viscoelasticity and of compressibility of the fluid in the reservoir yield oscillatory behaviors which can explain both the sharkskin texturing and spurt flow regime seen experimentally.

We thank Ken Elder and Rejean Ducharme for their collaboration in earlier stages of this work. This work was supported by the Natural Sciences and Engineering Research Council of Canada, and les Fonds pour la Formation de Chercheurs et l'Aide à la Recherche de Québec.

[1] M. M. Denn, Annu. Rev. Fluid Mech. 22, 13 (1990).

[2] S. G. Hatzikiriakos and J. M. Dealy, J. Rheol. 36, 845 (1992).

[3] P. G. de Gennes and C. R. Seances, Acad. Sci. Ser. B 288, 219 (1979); F. Brochard and P. G. de Gennes, Langmuir 8, 3033 (1992); F. Brochard-Wyart, P. G. de Gennes, H. Hervert, and C. Redon, ibid. 10, 1566 (1994).

[4] P. P. Drda and S.-Q. Wang, Phys. Rev. Lett. 75, 2698 (1995); Y. W. Inn and S.-Q. Wang, ibid. 76, 467 (1996).

[5] K. B. Migler, H. Hervet, and L. Leger, Phys. Rev. Lett. 70, 287 (1993); K. B. Migler, G. Massey, H. Hervet, and L. Leger, J. Phys. Condens. Matter 6, A301 (1994).

[6] J. Tatibouët, R. Gendron, and L. Piché (to be published).

[7] P. C. Hohenberg and B. I. Halperin, Rev. Mod. Phys. 49, 435 (1977); J. D. Gunton, M. San Miguel, and P. Sahni, in Phase Transitions and Critical Phenomena, Vol. 8, edited by C. Domb and J. L. Lebowitz (Academic Press, London, 1983), p. 267. For simplicity, we neglect thermal noise.

[8] A full description of local polymer conformation would involve a complicated order parameter and would be applied throughout the fluid, with its local expectation value related to shear thinning; for related ideas, see M. Urbakh, L. Dalkhin, and J. Klafter, J. Chem. Phys. 103, 10707 (1995), and references therein. However, here we consider only that aspect of the conformation which is relevant in determining the slipping length.

[9] G. C. Georgiou and M. J. Crochet, J. Rheol. 38, 639 (1994); 38, 1745 (1994). These authors find oscillatory behavior in the capillary flow of a compressible, Newtonian fluid, after assuming a nonlinear slip condition at the walls which gives rise to a multivalued flow curve.

[10] R. B. Bird, R. C. Armstrong, and O. Hassager, Dynamics of Polymeric Liquids, Vol. 1, Fluid Mechanics (Wiley, New York, 1987), 2nd ed.

[11] Using more complicated constitutive relations, it is possible to produce multivalued flow curves without assuming a stick-slip transition (see [1] for references). However, here we are motivated by the experimental evidence [5] that such a transition does in fact occur within microscopic distances of the capillary wall.

[12] J. D. Shore, D. Ronis, L. Piché, and M. Grant (to be published).

[13] Variations of the FitzHugh-Nagumo model have been used to describe, e.g., spiral waves in chemical reactions and neural excitations. [See A. Karma, Phys. Rev. Lett. 68, 397 (1992); A. T. Winfree, Chaos 1, 303 (1991).]

[14] W. H. Press, S. A. Teukolsky, W. T. Vetterling, and B.P. Flannery, Numerical Recipes in $C$ (Cambridge University Press, Cambridge, 1992), 2nd ed., Chap. 16.

[15] In experimental systems, $\xi_{0}$ should be roughly the characteristic size of a polymer; thus we expect $\xi_{0} \ll L_{x}$.

[16] See, e.g., P. Bergé, Y. Pomeau, and C. Vidal, Order within Chaos (Wiley, New York, 1984).

[17] The walls can behave symmetrically also, but this requires more than one roll across the channel, and so seems more difficult to create and sustain. 